\title{
Dorsal clitoral nerve injury following transobturator midurethral sling
}

\author{
Chailee F Moss' \\ Lynn A Damitz ${ }^{2}$ \\ Richard H Gracely ${ }^{3}$ \\ Alice C Mintz ${ }^{3}$ \\ Denniz A Zolnoun ${ }^{2-4}$ \\ A Lee Dellon ${ }^{5}$ \\ 'Department of Obstetrics and \\ Gynecology, Ohio State University \\ School of Medicine, Columbus, \\ OH, USA; ${ }^{2}$ Department of Surgery, \\ University of North Carolina at \\ Chapel Hill, NC, USA; ${ }^{3}$ Department \\ of Endodontics, University of \\ North Carolina at Chapel Hill, NC, \\ USA; ${ }^{4}$ Department of Obstetrics \\ and Gynecology, University of \\ North Carolina at Chapel Hill \\ School of Medicine, Chapel Hill, \\ NC, USA; ${ }^{5}$ Department of Plastic \\ and Reconstructive Surgery, Johns \\ Hopkins University School of \\ Medicine, Baltimore, MD, USA
}

This article was published in the following Dove Press journal: Journal of Pain Research

23 September 2016

Number of times this article has been viewed
Introduction: Transobturator slings can be successfully used to treat stress urinary incontinence and improve quality of life through a minimally invasive vaginal approach. Persistent postoperative pain can occur and pose diagnostic and therapeutic dilemmas. Following a sling procedure, a patient complained of pinching clitoral and perineal pain. Her symptoms of localized clitoral pinching and pain became generalized over the ensuing years, eventually encompassing the entire left vulvovaginal region.

Aim: The aim of this study was to highlight the clinical utility of conventional pain management techniques used for the evaluation and management of patients with postoperative pain following pelvic surgery.

Methods: We described a prototypical patient with persistent pain in and around the clitoral region complicating the clinical course of an otherwise successful sling procedure. We specifically discussed the utility of bedside sensory assessment techniques and selective nerve blocks in the evaluation and management of this prototypical patient.

Results: Neurosensory assessments and a selective nerve block enabled us to trace the source of the patient's pain to nerve entrapment along the dorsal nerve of the clitoris. We then utilized a nerve stimulator-guided hydrodissection technique to release the scar contracture

Conclusion: This case demonstrates that the dorsal nerve of the clitoris is vulnerable to injury directly and/or indirectly. Assimilation of a time-honored pain management construct for the evaluation and management of patients' pain may improve outcomes while obviating the need for invasive surgery.

Keywords: postoperative pain, complication, clitoral pain, nerve entrapment

\section{Introduction}

Upward of $9 \%$ of pelvic reconstructive procedures are complicated by postoperative pain. ${ }^{1}$ The anatomic course of several peripheral nerves in the pelvis (eg, pudendal, genitofemoral, ilioinguinal) are known. ${ }^{2}$ However, corresponding sensory landmarks in women are not well described nor are clinical signs and symptoms suggestive of postoperative pelvic neuropathy and its respective management. ${ }^{1}$ We present a case of peripheral neuropathy related to trauma to the dorsal nerve of the clitoris (DNC) during a transobturator sling procedure.

\section{Case presentation}

A 51-year-old woman presented with a 3-year history of pain described as pinching localized to her clitoris with radiation along her left medial thigh, which commenced
Correspondence: Denniz A Zolnoun Department of Obstetrics and Gynecology, University of North Carolina at Chapel Hill School of Medicine, Old Clinic Building Rm 4009, Campus Box 7570, Chapel Hill, NC 27599, USA

$\mathrm{Tel}+\mathrm{I} 9199668202$

Fax + I 9199665833

Email denniz_zolnoun@med.unc.edu 
following an anterior and posterior repair and transobturator sling placement for stress urinary incontinence (SUI). At the time of presentation, she was experiencing intractable left clitoral, groin, and vaginal pain; intimacy and movement caused radiating pain in her left lower abdomen, legs, and ankles. The patient gave written informed consent to be included in the study as well as for the use of the photos.

Previous treatments including physical therapy with vaginal dilators, amitriptyline, bupropion, gabapentin, and a ganglion impar block had been proved ineffective. At presentation, she was taking topiramate, which decreased the frequency and intensity of her episodic pain.

Examination revealed abdominal pinprick hyperalgesia, diminished light brushing sensation along the ilioinguinal nerve, and normal pelvic muscle movement. (Note: a detailed description of neurosensory assessment techniques will be presented in future manuscripts.)

Urogenital examination showed mechanical allodynia with a delayed onset of burning sensation. For the purpose of the aforementioned examination, the examiner gently moved a lubricated cotton swab transvaginally along the right and left paravaginal space. Gentle downward posterior vaginal traction caused shooting pain along the left labia minora and clitoris, followed by an aching pain generalized to the lower abdomen. Collectively, these findings along with other elements of the patient's history and examination suggested entrapment neuropathy that was peripherally maintained and likely related to scar contracture. To confirm this diagnosis, we proceeded with a selective nerve block and utilized a process of elimination to determine which nerve was the primary cause of the patient's pain. Doing so, we were able to conclude that DNC was the nerve causing the majority of the patient's pain.

In the absence of a selective nerve block, the patient's poor two-point discrimination and the convergence of sensory input from these peripheral nerves illustrated the presence of a significant overlap in symptom profiles.

We blocked the ascending perineal branches of the perineal nerve (Figure 1). Anatomical boundaries of this region can best be described in terms of its boundaries: superiorly confined to the space below an imaginary line drawn right to left across the external urinary meatus, laterally by the medial fold of the labia minora (including the vestibule), and medially by the hymenal ring.

Using anatomical landmarks, we performed a diagnostic nerve block by infiltrating $5 \mathrm{~cm}^{3}$ (1\% lidocaine) at the anatomical location of the ascending perineal nerve. This

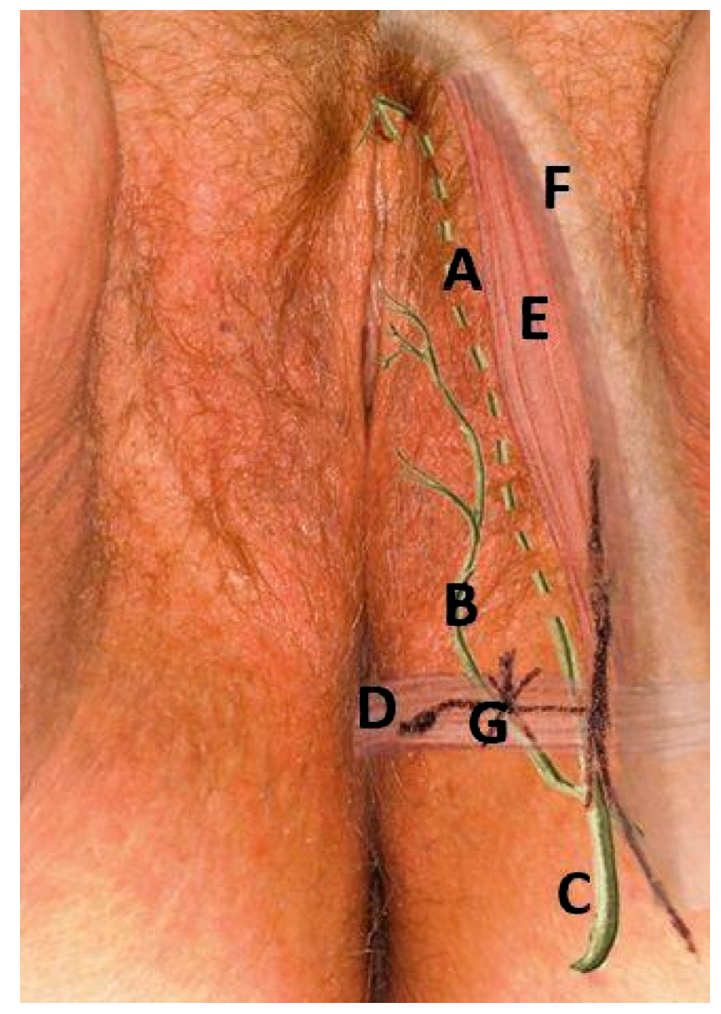

Figure I Pudendal branches, mapped by clinical exam and neurostimulation.

Notes: (A) Dorsal nerve of the clitoris (continuation of pudendal nerve shown as dashed line deeper in the muscles of the urogenital diaphragm); (B) perineal branch of the pudendal nerve; (C) pudendal nerve; (D) superficial transverse perineal muscle; (E) ischiocavernosus muscle; $(\mathbf{F})$ inferior pubic ramus; $(\mathbf{G})$ block location. The course of the specified nerves is delineated based on quantitative sensory testing and selective nerve blocks in this patient. The arrow delineates the site of needle entry for the selective block for the perineal branch of the pudendal nerve.

led to a $30 \%$ reduction in pain as reported by the patient. We subsequently expanded the field blocked by injecting the nerve at and around its anatomic division into DNC. A larger volume $\left(10 \mathrm{~cm}^{3}\right)$ was used to compensate for the lack of precision. Afterwards, the patient reported that over $60 \%$ of her pain was resolved, and one could palpate the exquisitely tender space at the level of the pubic rami and along the arms of the mesh on the left side.

During a subsequent visit, $4 \mathrm{~cm}^{3}$ of lidocaine injected into and above the region left of the clitoris (Figure 1) resulted in an immediate relief of the patient's symptoms. Administration of an additional $6 \mathrm{~cm}^{3}$ of the injection along the proximal clitoral nerve through the urogenital diaphragm eliminated mechanical allodynia and pelvic pressure sensitivity. The patient reported complete relief for 6-8 hours and a gradual return of symptoms within weeks. Because her symptoms returned slowly over time, the probable mechanism of her pain was likely related to entrapment. The likely mechanism given that the patient experienced immediate symptoms is initial traction/entrapment in the course of the procedure followed by additional scarring over time. 
Following the visit, the patient began tapering the bupropion and topiramate doses due to their side effects and lack of efficacy. She began a slow titration of duloxetine and trazodone to decrease central sensitization and improve her sleep as part of adjuvant medical management of her chronic pain.

A nerve stimulator-guided technique was subsequently used to localize the primary nerve (prior to arberization) at its exit site using an $80 \mathrm{~cm}$ needle that was bent to access the lateral region beyond the pubic rami. Nerve localization was confirmed by the patient's cues. At the site of maximal paresthesia, $2 \mathrm{~cm}^{3}$ of $1 \%$ lidocaine was injected, which led to an immediate improvement in the patient's pain. Saline was injected into the area of the scar, which was manually stretched to release a suspected nerve entrapment. ${ }^{3}$ Afterward, the patient experienced a sustained recovery; she continues to use centrally active medication and reported an $80 \%$ improvement 2 years later.

\section{Discussion}

Midurethral transobturator slings have high long-term success in the management of $\mathrm{SUI}^{4}$ and have been characterized as positively influencing sexual function as a result of reducing SUI. However, previous case reports indicated that sling procedures can cause damage to the pudendal, ilioinguinal, iliohypogastric, and genitofemoral nerves. ${ }^{2}$ Treatment of postoperative pain in DNC has not been reported.

We suspected that the mechanism of our patient's original pain was entrapment and anatomical distortion along the distal course of the pudendal nerve (Figure 1). This area is an anatomic watershed and the point of termination of the peripheral nerves originating from the lumbar (ilioinguinal and genitofemoral), perineal, and clitoral branches of the pudendal nerve.

DNC injury below the pubic bone is predicted during the placement of tension-free vaginal tape and during the dissection used in transobturator tape placement. ${ }^{2,5} \mathrm{DNC}$ is located along the internal aspect of the inferior pubic ramus inside Alcock's canal. DNC branches from the distal pudendal nerve then crosses over the pubic rami. ${ }^{5}$ However, in this case, the pain that the patient experienced with the application of pressure at the juncture of the inferior pubic ramus and the symphysis suggested a more anterior DNC location vulnerable to injury. ${ }^{6}$

A cadaveric study of postmortem sling placements with anatomic dissection of perineal structures predicted rarity of pudendal nerve branch injury. ${ }^{7}$ However, environmental factors (eg, childbirth) and anatomic variations (eg, varied pudendal nerve branching) may explain sporadic injury during sling procedures. ${ }^{6,8}$

Early injury recognition and prompt decompression are critical for the preservation of the nerves. Hydrodissection has been useful in cases of neurologic compression following carpal tunnel surgery, and it greatly aided our patient. ${ }^{9}$ Clinical training in neurologic examination of the pelvis and perineum is rarely provided in pelvic surgery or plastic surgery fellowships. This lack of training makes the diagnosis of acute iatrogenic nerve injury unlikely in the immediate postoperative period when early intervention is important for improving outcome. ${ }^{8,10,11}$ Our team recognizes that this manuscript does not provide sufficient detail for rapid dissemination and clinical adaptation. Our primary objective was to highlight the clinical implications of our collective research and development efforts to date in order to lay the foundation for future technique- and method-specific manuscripts.

Lastly, in most instances, we use concurrent medical management. As illustrated in this case, duloxetine and other centrally acting agents improve central sensitization in neuropathic pain, while local anesthetics may specifically address the peripheral component. ${ }^{1}$

\section{Conclusion}

This case demonstrates that careful symptomatic management with attention to isolating the peripheral versus the central component of pain may be effective in the evaluation and management of persistent postoperative pain following surgery. It also affirms the vulnerability of DNC during sling placement. This is an area where interventional peripheral nerve (ie, selective nerve block) and microsurgical expertise is lacking but extremely valuable.

\section{Acknowledgement}

This work, in part, was supported by the following NIH grants: P01NS045685 (Maixner/Zolnoun) and R01HD072983 (Gracely).

\section{Disclosure}

The authors report no conflicts of interest in this work.

\section{References}

1. Parnell BA, Johnson EA, Zolnoun DA. Genitofemoral and perineal neuralgia after transobturator midurethral sling. Obstet Gynecol. 2012; 119(2 Pt 2):428-431. 
2. Fisher HW, Lotze PM. Nerve injury locations during retropubic sling procedures. Int Urogynecol J. 2011;22(4):439-441.

3. Nieto ML, Brueseke T, Wu C, Geller E, Zolnoun D. Novel classification of labia anatomy in the evaluation and treatment of vaginal agglutination. Am J Clin Exp Obstet Gynecol. 2016;214(Suppl 4):S515.

4. Bekker M, Beck J, Putter H, et al. Sexual function improvement following surgery for stress incontinence: the relevance of coital incontinence. J Sex Med. 2009;6(11):3208-3213.

5. Achtari C, McKenzie BJ, Hiscock R, et al. Anatomical study of the obturator foramen and dorsal nerve of the clitoris and their relationship to minimally invasive slings. Int Urogynecol J Pelvic Floor Dysfunct. 2006;17(4):330-334.

6. Furtmuller GJ, McKenna CA, Ebmer J, Dellon AL. Pudendal nerve 3-dimensional illustration gives insight into surgical approaches. Ann Plast Surg. 2014;73(6):670-678.
7. Bonnet P, Waltregny D, Reul O, de Leval J. Transobturator vaginal tape inside out for the surgical treatment of female stress urinary incontinence: anatomical considerations. J Urol. 2005;173(4):1223-1228.

8. Hruby S, Dellon L, Ebmer J, Höltl W, Aszmann OC. Sensory recovery after decompression of the distal pudendal nerve: anatomical review and quantitative neurosensory data of a prospective clinical study. Microsurgery. 2009;29(4):270-274.

9. Malone DG, Clark TB, Wei N. Ultrasound-guided percutaneous injection, hydrodissection, and fenestration for carpal tunnel syndrome: description of a new technique. J Appl Res. 2010;10:116-123.

10. Tu FF, Hellman KM, Backonja MM. Gynecologic management of neuropathic pain. Am J Obstet Gynecol. 2011;205(5):435-443.

11. Dellon AL, Coady D, Harris D. Pelvic pain of pudendal nerve origin: surgical outcomes and learning curve lessons. J Reconstr Microsurg. 2015;31(4):283-290.

\section{Publish your work in this journal}

The Journal of Pain Research is an international, peer reviewed, open access, online journal that welcomes laboratory and clinical findings in the fields of pain research and the prevention and management of pain. Original research, reviews, symposium reports, hypothesis formation and commentaries are all considered for publication.

\section{Dovepress}

The manuscript management system is completely online and includes a very quick and fair peer-review system, which is all easy to use. Visit http://www.dovepress.com/testimonials.php to read real quotes from published authors. 